\title{
Design on Energy-saving Mechanism of 6LoWPAN Wireless Sensor Net- work
}

\author{
Zhenfeng Qu ${ }^{*}$ and Xiaoguang Li
}

Department of Electrical Engineering and Automation, Luoyang Institute of Science and Technology, Luoyang, Henan, 471023, China

\begin{abstract}
Wireless sensor network LAN is mostly ad hoc form, and it only can access and process information in the dedicated control center. IP network is widely used, if such a local wireless sensor network access can be accessed to internet backbone network, it will bring users more convenience. This paper designs a new wireless sensor network of 6LoWPAN using IPv6 and IEEE802.15.4 protocol, and adopts the low duty and multi distributed IP to optimize the network, finally saving cyber source and reducing the network energy consumption in the process of sending, receiving data, which achieves a seamless mosaic of wireless sensor network and IP network. This paper uses solar cell technology to improve the terminal sensor of sensor network and reduce the energy consumption of sensor and processor. Finally, based on the testing of wireless network, 6LoWPAN wireless networks has a significant energy consumption decrease in each function module and information transmission environment, which provides a theoretical reference for the study on wireless sensor network energy saving mechanism.
\end{abstract}

Keywords: 6LoWPAN network, C language, Contiki integration, distributed IP, IEEE802.15.4.

\section{INTRODUCTION}

With the development of computer network technology, more and more devices need to access the network. The 6LoWPAN working group will be dedicated to apply the IPv6 in IEEE 802.15.4, so as to realize the seamless connection between wireless sensor network and IP network [1-3]. 6LoWPAN wireless sensor network formation is mainly related with network configuration and sensing terminal energy saving, and the energy saving is mainly through the network configuration and energy saving of wireless sensor terminal.

For the saving energy problem of sensor nodes, many experts and scholars have done a lot of research and have designed many ways to solve the problem $[4,5]$. But in general, the method of obtaining energy from nature is the most convenient and lasting effect, so solar energy is widely applied.

The hardware design is the main task of solar energy sensor nodes. Many domestic and foreign experts have carried on the design to the hardware and algorithm, the representative is Trio experiment system completed by the Dutta team [6-9]. The system uses power solar panels to replace the traditional supply model, and the network consists of solar powered general Trio nodes and gateway Trio nodes. Nodes provide continuous power through a large solar panel collecting environmental energy.
C. Park et al. have designed more perfect energy acquisition system, using super capacitor storage display and control charging module, and design the maximum power tracking algorithm, which can adjust the size of the capacitor voltage according to power supply mode. When the capacitor voltage is above a certain threshold value, the super capacitor supply power for sensor node; on the contrary, when less than the threshold, the battery will supply power [10-12].

In this paper, the aim is to provide a centrifugal apparatus, which can be applied in the industry. And, to further provide a means to develop the pressure sufficiently to extrude plastic resinous material without employing conventional extrusion equipment.

\section{LOW DUTY RATIO ENERGY SAVING MECHA- NISM FOR WIRELESS SENSOR NETWORK}

At present there are two terminal sensors solar battery to realize the design of low power consumption, including Mono-Crystal silicon and polycrystalline silicon solar cell [13]. Single crystal silicon solar cell is based on single crystal silicon as its basic structure as shown in Fig. (1).

Single crystal silicon cell has stable generation performance, and it is one of the largest used solar cells. Compared with polycrystalline silicon battery, the solar energy conversion efficiency is high, but the production has high purity requirements for raw materials, so the cost of production is higher. Compared with Mono-Crystal silicon solar energy cell, the advantage of polysilicon solar cells is saving materi$\mathrm{al}$, and the disadvantage is the low conversion efficiency [14]. 

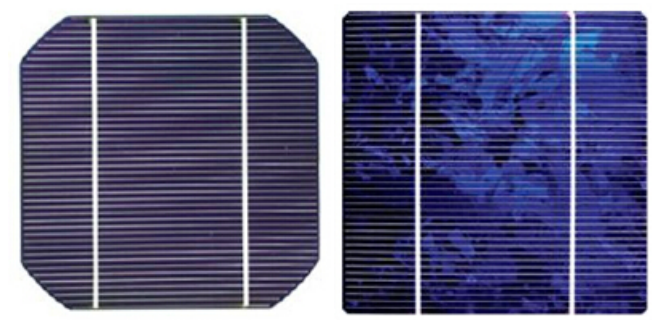

Fig. (1). Mono-crystal silicon solar cell.

In general, traditional sensor networks basically let the nodes in the whole state, that is to say the node have been waiting to receive data and listen if there is data sent over [15]. But it will waste a lot of useless energy, because the actual data transmission time in the wireless sensor network node is not long, most of the time they are idle listening. Some low power chip, such as CC2430 series design by TI, they have the dormancy and listening mode and allows nodes alternately in the two modes, so in idle listening state it can let the node sleep and reduce the overall energy consumption.

Compared with the traditional sensor networks, in order to make the network life stronger, the domestic and foreign scholars apply the low duty ratio MAC mechanism in wireless sensor network, later known as the low duty ratio sensor network [16]. For this wireless sensor network mechanism, when data arrives, it can periodically wakes up and receive data, the rest of the time it is in a dormant state. Therefore, this mechanism can obviously prolong the network life cycle.

\section{6LOWPAN NETWORK ENERGY SAVING MECH- ANISM AND ALGORITHM DESIGN}

The establishment of wireless sensor energy saving mechanism can greatly reduce energy consumption and save computer resource and energy expenditure, which has the vital significance for the establishment of wireless sensor network. This paper is mainly based on the principle of wireless sensor energy consumption, combined with low duty ratio and multiple distributed network system to optimize wireless sensor network, and establishes the optimization model of wireless network energy consumption, and improves the efficiency of the network transmit and receive data through the design of distributed network, achieving the overall implementation of energy-saving effect.

\subsection{LoWPAN Wireless Sensor Network Energy Con- sumption and Duty Ratio Optimal Design}

In order to improve the transmission efficiency of 6LoWPAN, the wireless network communication line uses optical fiber transmission, and the network signal consuming in the optical fiber line is mainly related to optical system loss and dispersion. The optical function loss maximum effective transmission distance is as shown in equation (1).

$g=\frac{G_{m}-G_{n}-G_{c}-2 K_{p}-L_{p}}{\lambda_{a}-\lambda_{b}}$
Where, $G_{m}$ is luminous power when completely loss; $G_{n}$ is receive sensitivity with no safety loss; $Y_{n}$ is the luminous flux rate; $\lambda_{a}$ and $\lambda_{b}$ respectively are the light loss coefficient; $K_{p}$ is loss of the cable interface; $L_{p}$ is the maximum rate of cable communication. In the 6LoWPAN in wireless sensor networks, duty ratio is the key of energy consumption. This paper adopts IEEE802.15.4 transmission protocol, and it can optimize the duty ratio. Assume that there are $K$ duty ratios for $Y$ layers as: $Y_{1}, Y_{2}, \cdots, Y_{k}$, so the overall goal of $X$ order is $y_{1}, y_{2}, \cdots, y_{k}$. The hierarchical ranking of monolayer is as equation (2).

$g=\frac{G_{m}-G_{n}-G_{c}-2 K_{p}-L_{p}}{\lambda_{a}-\lambda_{b}}$

The total order of $N$ layer is:

$$
\begin{aligned}
& N_{1}: y_{1} z_{11}+y_{2} z_{12}+\cdots y_{k} z_{1 l} \\
& N_{2}: y_{1} z_{21}+y_{2} z_{22}+\cdots y_{k} z_{2 l} \mathrm{c} \\
& \cdots \\
& N_{l}: y_{1} z_{k 1}+y_{2} z_{k 2}+\cdots y_{k} z_{k l}
\end{aligned}
$$

The ratio of $N$ layers $j$ factors for the whole annulus frame structure is:

$$
\sum_{i=1}^{l} y_{i} z_{j i}
$$

In order to optimize the loop control ratio and make it optimal in the network transmission process, it needs overall consistency optimization. If single level optimization coefficient is $K L_{i}$, random consistency optimization coefficient is $G L_{j}$, so the overall optimization coefficient is:

$$
Z Y=\frac{m_{1} K L_{1}+m_{2} K L_{2}+\cdots+m_{k} K L_{k}}{m_{1} G L_{1}+m_{2} G L_{2}+\cdots+m_{m} G L_{m}}
$$

When $Z Y<0.1$, 6LoWPAN wireless network duty ratio is optimal. If the overall is poor, it needs to re-adjust to get the new duty ratio optimization proportion. 


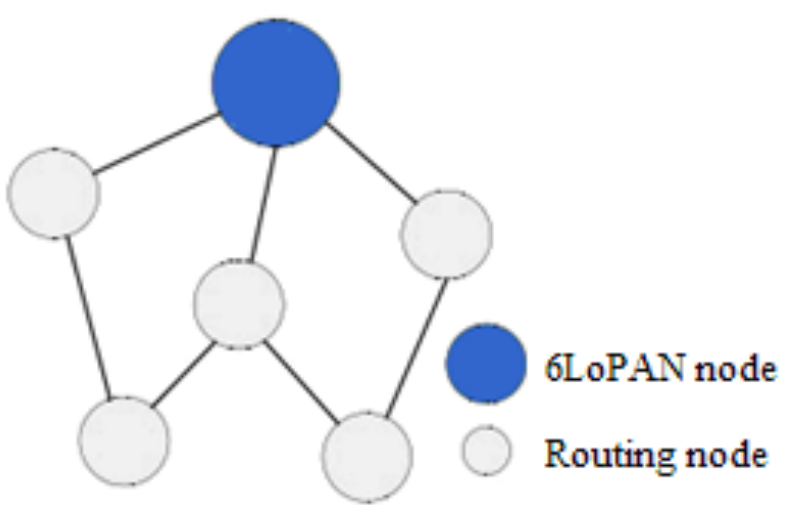

Fig. (2). Schematic model of IPv6 wireless sensor network node.

\subsection{Mesh Structure Energy-saving Distribution Design for 6LoWPAN Wireless Sensor Network}

Distributed network is a new kind of network architecture model in recent years. In the network, nodes can directly transmit information, and it can choose the better bearing ability node as a 6LoWPAN node in many nodes, completing information exchange task and saving the network resources.

As shown in Fig. (2), distributed IPv6 network can greatly improve the utilization rate of network nodes and form a wide coverage range of wireless network, which effectively reduces the energy consumption. And it can be applied in network energy saving mechanism of 6LoWPAN. Distributed network control principle is mainly based on the network nodes to form network 3D model, and it can use independent node in 6LoWPAN to establish a local network space, and the model is shown in equation (6).

$S=a+b x+c y+d z$

Wireless network nodes can use formal and real method to meet the calculation needs of $3 \mathrm{D}$ space function. In equation (6), $x$ represents the real part of $S, b x+c y+d z$ represents the imaginary part of $S$, and then $S$ can be done binary random coding as shown in equation (7).

$\left\{a_{t-1}, s_{t-1}^{1}, s_{t-1}^{2}, \cdots, s_{t-1}^{n}\right\}, a_{t-1} \in R$

Each network node $\left\{S_{e}^{1}, S_{e}^{2}, \cdots, S_{e}^{n}\right\}$ and $\left\{S_{f}^{1}, S_{f}^{2}, \cdots, S_{f}^{n}\right\}$ are done spatial crossover and mutation copy operation as shown in equation (8).

$\bar{S}_{e}^{j}=x S_{e}^{j}+(1-x) S_{e}^{j}$

$\bar{S}_{f}^{j}=(1-x) S_{f}^{j}+x S_{f}^{j}, j=1,2, \ldots, n$

Where, $x$ is a random number and $x \in[0,1]$, using repeated calculation of crossover and mutation operation, it can reach the local and global optimization of distributed network.
3.3. 6LoWPAN Network Integrated Environment Programming Design

This section uses Contiki studio integrated development platform to design the integrated network environment, and uses $\mathrm{C}$ language to develop code, first define an Contiki process. Its main code is as follows:

\#include "contiki.h"

\#include $<$ stdio.h $>$

PROCESS(hello_world_process,"Hello world");//PROCESS(name,strname)

AUTO-

START_PROCESSS(\&hello_world_process);//AUTOSTAR T_PROC̄ESS $(\ldots)$

PRO-

CESS THREAD(hello world process,ev,data)//PROCESS THREAD(name,ev, data)

\{

PROCESS_BEGIN();

$/ * * \operatorname{code} * * /$

PROCESS_END();//The main function of the process ends from here

After setting the Contiki process, it needs to develop API program of Contiki and the application program interface, and its main procedure is as follows:

- The process declaration and definition:

PROCESS_THREAD(name, ev, data)

PROCESS_NAME(name)

PROCESS(name, strname)

- $\quad$ Process protothread method

PROCESS_BEGIN()

PROCESS END()

PROCESS_EXIT()

PROCESS_CURRENT()

PROCESS_CONTEXT_BEGIN(p)

PROCESS_CONTEXT_END(p)

PROCESS_POLLHANDLER(handler)

\section{EXPERIMENTAL STUDY ON 6LOWPAN WIRE- LESS SENSOR NETWORK SET UP AND ENERGY SAVING MECHANISM}

In order to verify the effectiveness and reliability of 6LoWPAN wireless sensor network energy-saving mechanism designed in section second, this paper will combine the 


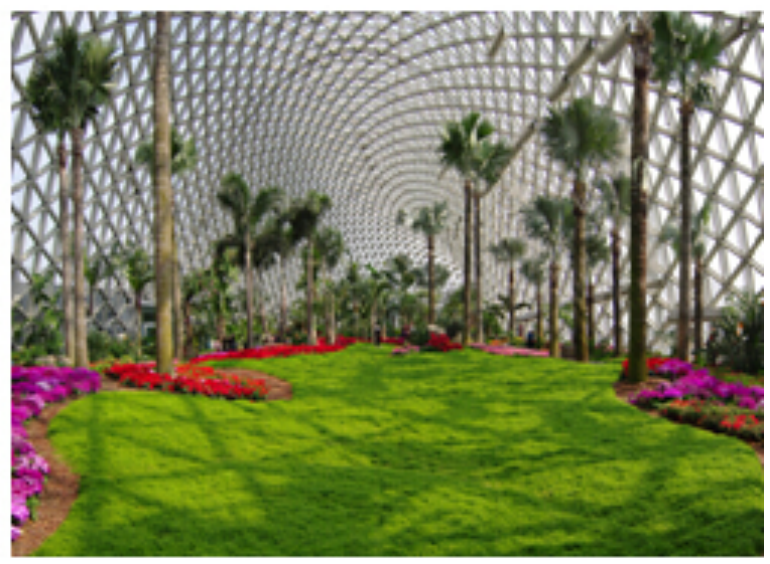

Fig.(3). Schematic diagram of greenhouse sensor network.

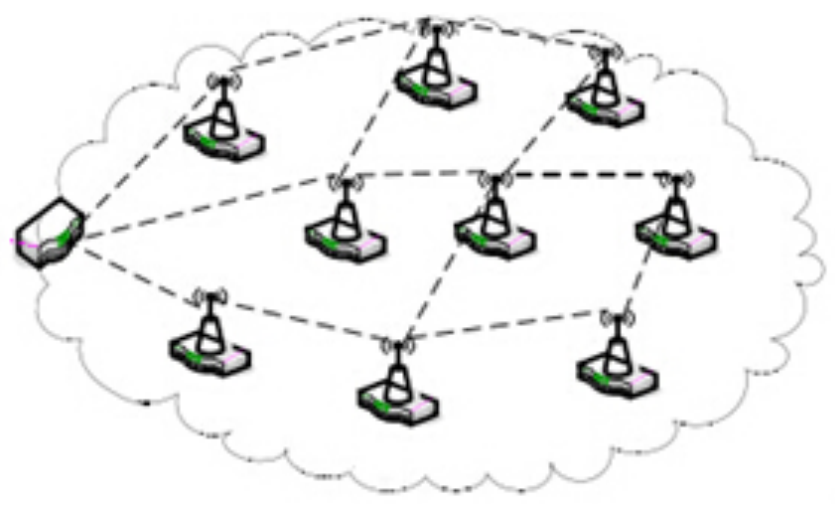

Fig. (4). Multi distributed wireless sensor network layout.

wireless sensor network and IPv6, and introduces IEEE802.15.4 technology into the network design, and does seamless splicing experiment on wireless sensor network and IP network.

\subsection{LoWPAN Wireless Sensor Network Layout}

For sensor networks distribution, this paper adopts distributed structure and multi sensor terminal form, and uses sensor node and gateway equipment to layout wireless sensor network. The diagram of sensor layout environment is as shown in Fig. (3).

Fig. (3) shows the schematic diagram of wireless sensor network erects environment. In order to fully verify the energy saving effect of solar sensor terminal, this paper chose the better sunlight greenhouse flowers as the experimental greenhouse, and uses the multi distributed structure as the network laying as shown in Fig. (4).

\subsection{The Integrated Development Environment of 6LoWPAN Wireless Network}

The experiment uses suite developed by Contiki platform, which supports IEEE802.15.4 physical transmission protocol and IPv6 development environment. The hardware devices are as shown in Fig. (5).
Fig. (5) shows the experimental hardware equipment of 6LoWPAN wireless sensor network designed in this paper. The hardware part is mainly composed of transmission nodes, transmission module and transmission gateway. The software part is mainly composed of a Contikistudio integrated development platform. Software integration environment installation is as shown in Fig. (6).

Fig. (6) shows Contiki uses C language to develop program. It has lower hardware requirements and is suitable for use in a variety of micro computer and the computer, and can be used in PC single chip. Based on the 6LoWPAN wireless network, this paper developed the data transmission procedure, wherein the program of sensor terminal obtaining data has been given in second part.

\subsection{Energy Saving Mechanism Testing in 6LoWPAN Wireless Sensor Network}

In order to test the 6LoWPAN energy conservation mechanism designed in this paper, this paper uses Firefox browser to test the system. The system consists of browser, web server, IPv6 intelligent gateway, MX231CC nodes. In order to verify the effect of network energy saving, this section studies the characteristics of network mainly from the energy consumption. 


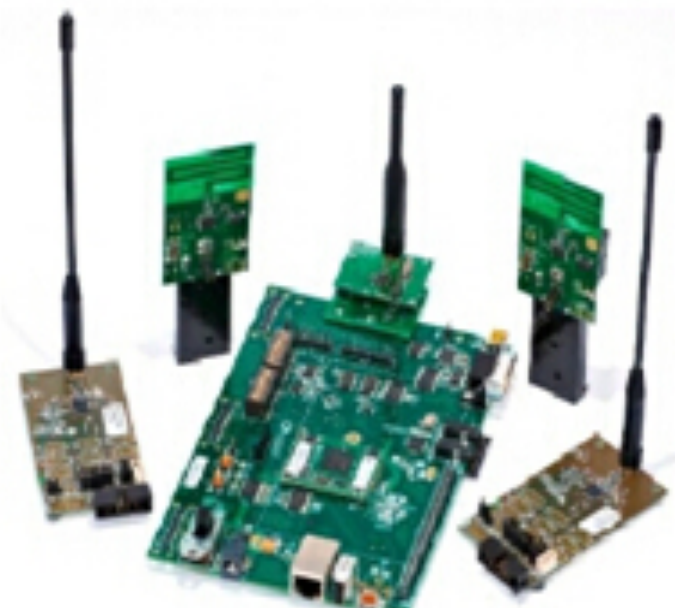

Fig. (5). The experimental hardware equipment diagram.

\begin{tabular}{|c|c|c|}
\hline \multicolumn{2}{|l|}{ Register } & $x$ \\
\hline \multicolumn{2}{|c|}{ Power Mgmt and Clocks $\quad \checkmark$} & \\
\hline 円SLEEPSTA & $=0 \times 40$ & \\
\hline 团SLEEPCMD & $=6 \times 04$ & \\
\hline 円CLKCONSTA & $=0 \times B 0$ & \\
\hline 曰CLLCONCMD & $=0 \times B 0$ & \\
\hline$-0 s c 32 \mathrm{~K}$ & $=1$ & \\
\hline- osc & $=0$ & \\
\hline - TICKSPD & $=0 \times 6$ & \\
\hline L CLKSPD & $=0 \times 0$ & \\
\hline$\boxplus P C O N$ & $=0 \times 00$ & \\
\hline$\boxplus$ ĐLD & $=0 \times 00$ & \\
\hline
\end{tabular}

Fig. (6). The contiki studio integrated development.

\section{Current/mA}

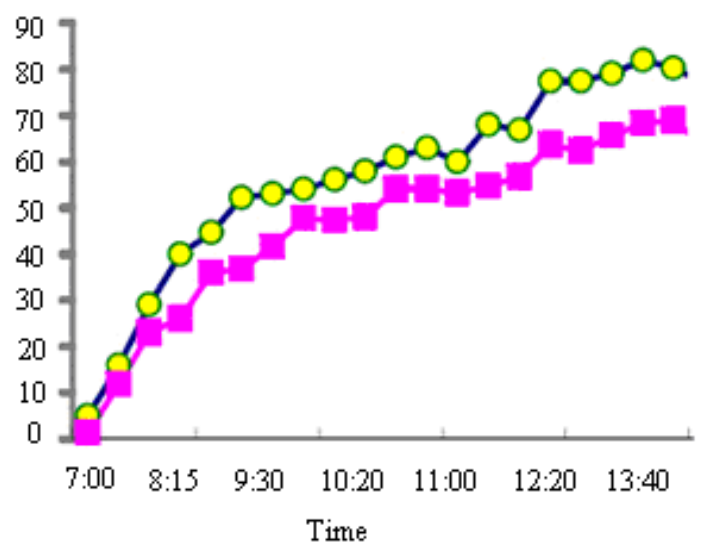

Fig. (7). The current comparison curves of 6LoWPAN and the general network.

Fig. (7) shows the current contrast curve of 6LoWPAN and the general network. From the chart it can be seen, in the working process, the current change tendency is the same, but the current of 6LoWPAN wireless sensor network is obviously smaller, thereby reducing the energy consumption of wireless sensor network.
Fig. (8) shows the energy saving effect of 6LoWPAN wireless sensor network in different modules and different conditions. No.1 and No.2 represent the network sensing terminal solar energy saving and processor energy saving percentage; No.3-6 respectively are energy-saving effect in receiving, sending, idle and sleep condition. From the 


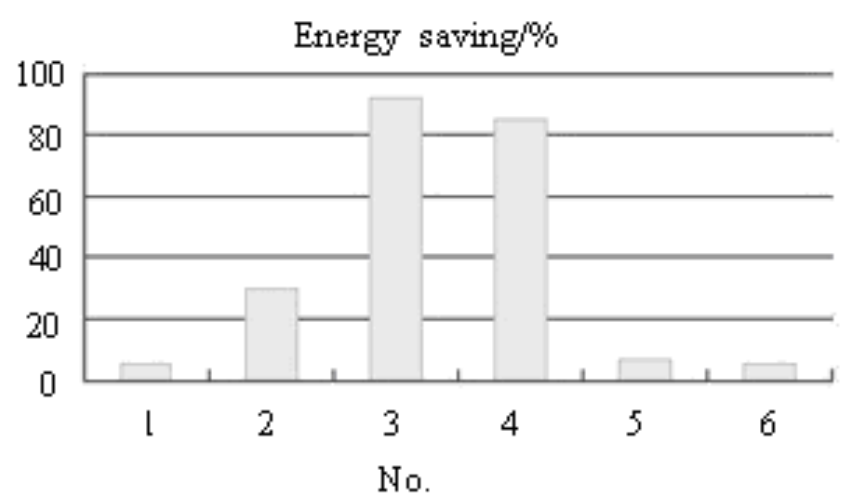

Fig. (8). Node of each unit energy saving.

Table 1. Energy saving statistical table of 6LoWPAN wireless sensor network.

\begin{tabular}{|c|c|c|}
\hline Testing No. & 6LoWPAN Sensor Network Energy Saving (mV) & Energy Saving (\%) \\
\hline \hline 1 & 1.12 & 5.0 \\
\hline 2 & 1.28 & 90 \\
\hline 3 & 9.25 & 85 \\
\hline 4 & 8.72 & 7.0 \\
\hline 5 & 0.70 & 5 \\
\hline 6 & 0.29 & 5 \\
\hline
\end{tabular}

Fig. (8), it can be seen, sensor terminal solar battery has better energy saving effect. The energy saving effects are different in the sending, receiving, idle and sleep states. The energy saving specific values is as shown in Table $\mathbf{1}$.

From the Table 1, it can be seen that energy consumption is the biggest when the nodes sends and receives data. When the device is idle, the monitoring channel is still in working condition, so that the energy consumption is relatively large, other times with relatively low energy consumption. From the table it also can be seen, the energy saving effect of 6 LoWPAN is very obvious, with maximum $92 \%$. From the energy saving effect, 6LoWPAN wireless sensor network has better energy saving effect, so it is promoted in the seamless splicing process of wireless network and IP network.

\section{CONCLUSION}

This paper designs multiple distributed IP mathematical model of 6LoWPAN network transmission energy consumption and low duty ratio, and the model is realized by using $\mathrm{C}$ language program, finally a new kind of wireless sensor network energy saving mechanism is obtained.

At the same time, this paper uses distributed IP technology, IEEE802.15.4 protocol, Contiki integrated development platform to build experimental platform of 6LoWPAN wireless sensor network energy saving mechanism, and uses the
Firefox browser and wireless network to verify the effect of energy saving network. Finally, through the experiment, energy consumption of 6LoWPAN wireless network has significantly reduced in each function module and information transmission environment, which saves the cyber source, including the solar sensor terminal, processor, sending and receiving information module and link, to provide a theoretical reference for the research on wireless sensor network energy saving mechanism.

\section{CONFLICT OF INTEREST}

The authors confirm that this article content has no conflict of interest.

\section{ACKNOWLEDGEMENTS}

Declared none.

\section{REFERENCES}

[1] X. N. Wang, and H.Y. Qian, "State address configuration scheme of 6LoWPAN wireless sensor network," Computer Application Research, vol. 3, pp. 35-36, 2012.

[2] Y.J. Wang, Z.H. Qian, X. Wang, and D.Y. Sun, "Internet of things addressing strategy based on 6LoWPAN," Journal of Electronics \& Information Technology, vol. 1, pp. 763-769, 2012. 
[3] X.K. Zhang, and E. J. Bai, "6LoWPAN sensor gateway design," Electronic Science and Technology, vol. 4, pp. 11-14, 2012.

[4] Z.J. Hong, H. Sun, M.Z. Li, F. Zhang and X.H. Li, "Development of a vehicle-mounted crop detection system," Journal of Integrative Agriculture, vol. 2, pp. 1284-1292, 2014.

[5] J.E. Su, S.X. Li, Y.X. Hu, D.X. Wang and X.M. Yin, "The mobile terminal controlling the intensive baking process based on internet of things," Electronic Technology and Software Engineering, vol. 1, pp. 254-256, 2014.

[6] S. W. Yang, X. E. "Application of IOT technology in food safety," Software, vol. 3, pp. 131-134, 2014.

[7] J.S. Song, Z.H. Xin, L.Y. Hu and B. Liu, "Design and realization intelligent dog based on internet of things," Journal of Suzhou University, vol. 7, pp. 90-92, 2014.

[8] Y.H. Zhu, H. Xu and J. Peng, "The two step index backoff algorithm for IEEE 802.11 wireless network," Electronic and Information Science, vol. 11, pp. 2575-2580, 2011.

[9] J.Y. Gu, G.A. Zhang and Z.H. Bao, "The cognitive wireless Mesh network multi path routing and channel allocation strategy," Computer Science, vol. 2, pp. 45-48, 2011.
[10] H. Zhong, and G.Z. Wang, "P2P model based on improved Pastry," Communication Technology, vol. 43, pp. 96-99, 2012.

[11] X.D. Fang, J. Zhang and X.R. Zhang, "The communication mechanism and network governance instant network era," Modern Communication, vol. 5, pp. 64-69, 2011.

[12] L.J. Chen, S.W. Mei and Y. Chen, "The impact of intelligent grid information security on the survival of power systems," Control Theory and Applications, vol. 29, pp. 240-244, 2012.

[13] J. Hu, "Strategy of strong smart grid," National Grid, vol. 1, pp. 25-28, 2011.

[14] S.Q. Zhang, Y.G. Sun, T. Yang, and Z. Cui, "Application of wireless sensor network in intelligent power grid," China Electric Power, vol. 43, pp. 31-36, 2012.

[15] X.P. Fan, Z.Y Xiong., Z.J. Chen, S.Q. Liu and Z.H. Qu, "Research on wireless multimedia sensor network video coding," Journal of Communication, vol. 32, pp. 137-146, 2011

[16] R.X Lu, and Z. Yu, "Research and implementation of J2EE platform and MVC design pattern," Application of Computer Application, vol. 20, pp. 144-146, 2011.

(C) Qu and Li; Licensee Bentham Open.

This is an open access article licensed under the terms of the Creative Commons Attribution Non-Commercial License (http://creativecommons.org/licenses/by$\mathrm{nc} / 3.0 /$ ) which permits unrestricted, non-commercial use, distribution and reproduction in any medium, provided the work is properly cited. 